\title{
Primary cutaneous CD4+ small/medium T-cell lymphoma: a case report
}

\author{
Jeenam Kim ${ }^{1}$, \\ Minkyoung Jeong ${ }^{1}$, \\ Dongkeun Jun ${ }^{1}$, \\ Myungchul Lee ${ }^{1}$, \\ Donghyeok Shin ${ }^{1}$, \\ Wookyoun Kim², \\ Hyungon Choi ${ }^{1}$ \\ Departments of ${ }^{1}$ Plastic and \\ Reconstructive Surgery and ${ }^{2}$ Pathology, \\ Konkuk University Medical Center, \\ Seoul, Korea
}

\begin{abstract}
Primary cutaneous CD4+ small/medium T-cell lymphoproliferative disorder is a rare disease characterized by a single mass on the face or upper part of the trunk. It usually presents an asymptomatic and favorable progression, and its histopathologic findings include small and medium-sized lymphoid cells. The authors report a case of primary cutaneous CD4+ small/medium T-cell lymphoproliferative disorder on the forehead. A 51-year-old man presented with a protruding mass on his forehead that the patient had noted 1 month previously. Surgical excision and a permanent biopsy were performed under local anesthesia. Based on the biopsy results, the mass was diagnosed as a primary cutaneous CD4+ small/medium T-cell lymphoproliferative disorder. There was no evidence of recurrence at a 15-month follow-up visit.
\end{abstract}

Abbreviations: CD, cluster of differentiation; PET, positron emission tomography; TCR, T-cell receptor.

Keywords: CD4 / Lymphoma / Lymphoproliferative disorder

\section{INTRODUCTION}

Primary cutaneous CD4+ small/medium T-cell lymphoproliferative disorder was previously classified as primary cutaneous CD4+ small/medium pleomorphic T-cell lymphoma. In 2016, when the lymphoma classification was revised, the World Health Organization-European Organization for Research and Treatment of Cancer changed the term to primary cutaneous CD4+ small/medium T-cell lymphoproliferative disorder due to its uncertain potential for malignancy $[1,2]$. Clinically, it usually manifests as a single erythematous nodule or mass in the head and upper part of the body [3,4]. Histopathological findings show dense infiltration of small or medium lymphocytes [1-3]. The authors report a case of primary cutaneous CD4+ small/medium T-cell lymphoproliferative disorder in the forehead of a 51-year-old man.

\section{Correspondence: Hyungon Choi}

Department of Plastic and Reconstructive Surgery, Konkuk University Medical

Center, 120-1 Neungdong-ro, Gwangjin-gu, Seoul 05030, Korea

E-mail: 20040059@kuh.ac.kr

Received May 12, 2021 / Revised July 5, 2021 / Accepted August 11, 2021

\section{CASE REPORT}

A 51-year-old man visited our outpatient clinic with a protruding red mass on the forehead that he had noted 1 month previously. The mass was circular, with dimensions of $1.0 \times 1.0 \mathrm{~cm}$ and a clear boundary, and was generally firm to the touch (Fig. 1). The patient did not complain of pain, tenderness, or sensory abnormality caused by the mass. There was no lymph node hypertrophy or systemic symptoms, but the mass was gradually growing. The patient had no history of trauma, surgery, or medication, and no specific history was identified other than his father's family history of liver cancer. An excisional biopsy including normal skin was performed under local anesthesia. The defect was closed primarily. The safety margin was $5 \mathrm{~mm}$ on both sides, and $3 \mathrm{~mm}$ cranially and caudally. Histopathology showed that small or medium lymphocytes were distributed with mild pleomorphism. Immunostaining showed primary cutaneous CD4+ small/medium T-cell lymphoproliferative disorder, in which the tumor cells were positive for $\mathrm{CD} 4, \mathrm{CD} 3$, programmed death 1 , and inducible co-stimulator; negative for 
CD20 and CD30; 10\%-20\% positive for Ki-67; negative for Epstein-Barr virus; and negative for monoclonal B-cell proliferation and clonal T-cell receptor (TCR)-gamma gene rearrangement (Fig. 2). To confirm the above results, additional laboratory and imaging tests were conducted in collaboration with the hematology-oncology department to ensure proper diagnosis

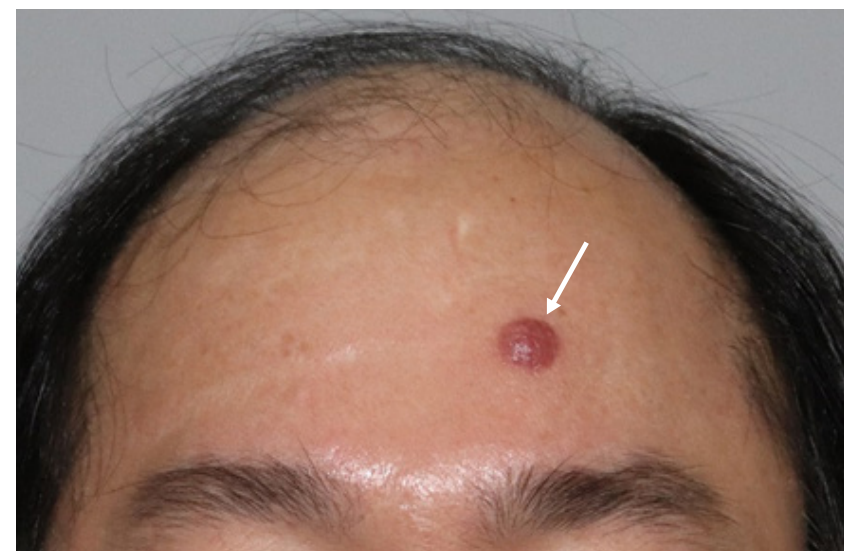

Fig. 1. Preoperative photograph showing an erythematous mass on the forehead (white arrow). and treatment. Laboratory tests were negative for human T-cell lymphotropic virus I/II antibodies. No specific findings were observed on additional computed tomography and positron emission tomography (PET) scans. On the PET scan, physiological muscular uptake in the left ventricle of the heart was identified, but not significant uptake (Fig. 3). No other complications, including recurrence, were observed except for minor scar formation at the surgical site during a 15-month postoperative follow-up (Fig. 4).

\section{DISCUSSION}

Primary cutaneous CD4+ small/medium T-cell lymphoproliferative disorder is a rare disease that accounts for 3\% of all primary cutaneous lymphomas; it most often occurs in patients in their 50s and 60s, but is occasionally found in children [1-5]. Most cases involve an asymptomatic, mono-nodular mass on the face, neck, or upper body of the trunk; however, this condition can infrequently occur in other areas, present as multiple nodules, or be accompanied by symptoms such as pain $[1,4,6-$ 8]. Immunohistochemical staining of lesions shows small and
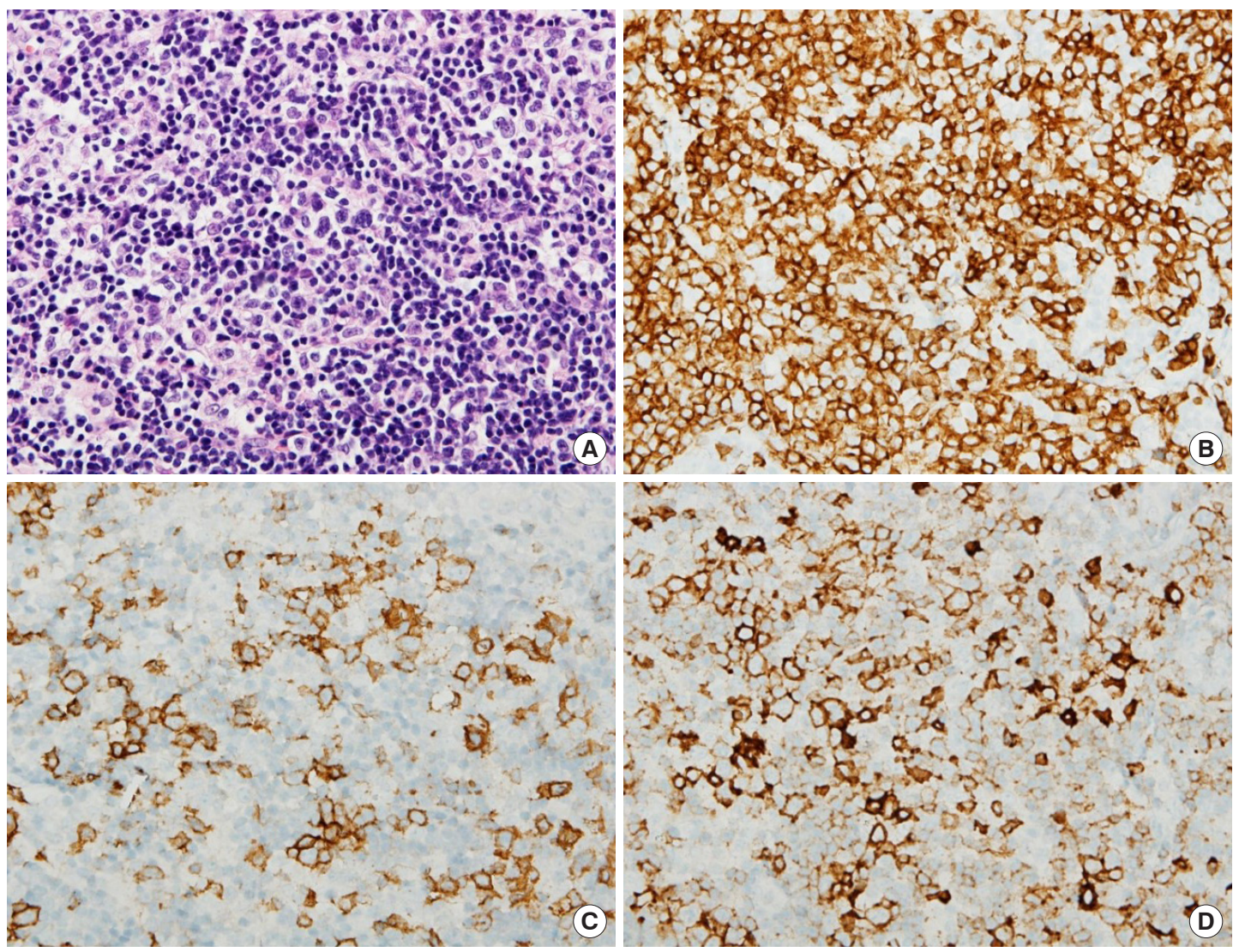

Fig. 2. Histopathology of the primary cutaneous CD4+ small/medium T-cell lymphoproliferative disorder. (A) The lymphoid cells are small to medium-sized with mild pleomorphism $(\mathrm{H} \& \mathrm{E}, \times 400)$. (B) The lymphoid cells mostly show the CD4+ phenotype $(\mathrm{CD} 4, \times 400)$. (C) The tumor cells are also positive for programmed death $1(\mathrm{PD}-1, \times 400)$ and $(\mathrm{D})$ inducible co-stimulator $(\mathrm{ICOS}, \times 400)$. 


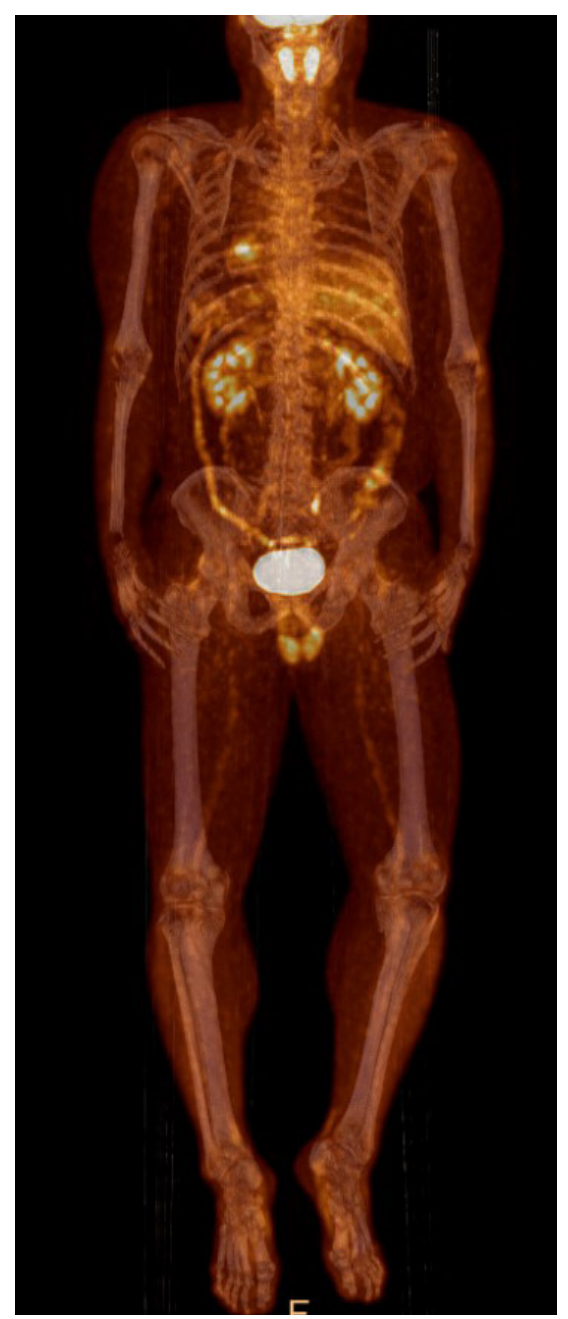

Fig. 3. Positron emission tomography showing no remarkable findings. Physiological muscular uptake in the left ventricle of the heart is present, but not significant uptake.

medium pleomorphic $\mathrm{T}$ cells on the dermis, positivity for $\mathrm{CD} 3$ and $\mathrm{CD} 4$, and negativity for CD8 and CD30 [6-8]. For an accurate diagnosis, it must be differentiated from other lymphoproliferative disorders with similar pathological, histological, and immunostaining results, necessitating a correlation between clinical findings and pathological results. The differential diagnosis includes cutaneous pseudolymphoma, cutaneous B-cell lymphoma, peripheral T-cell lymphoma, and mycosis fungoides $[2,4,7,9]$. Patients diagnosed with this disease based on a skin biopsy, receive laboratory tests (including a complete blood count and blood chemistry), human T-cell lymphotropic virus testing, TCR gene rearrangement testing, and computed tomography and PET scans $[1,2]$. The 5 -year survival rate is $60 \%$ to $80 \%$, and the prognosis is affected by the size of the tumor, the presence of multiple nodules, and differentiation [6,7]. Although there is no specific consensus regarding treatment, previous reports have suggested chemotherapy, radiation therapy,
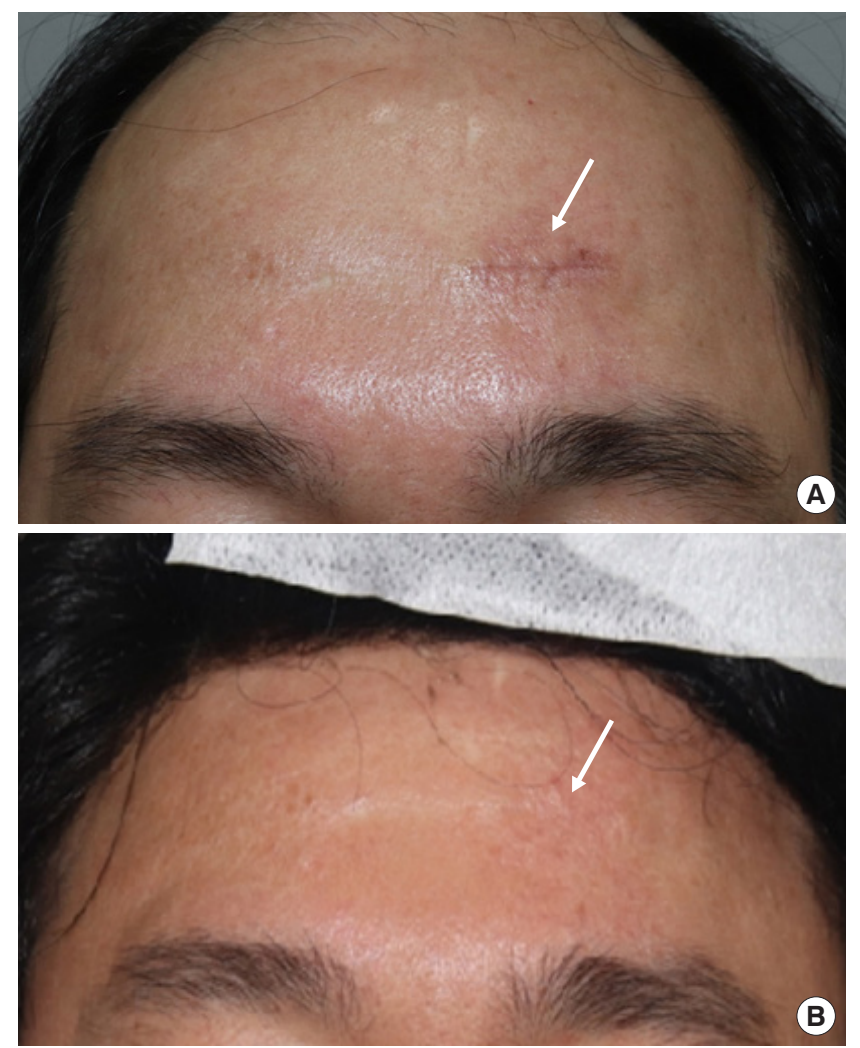

Fig. 4. Postoperative photographs. (A) One-month postoperative photograph showing a 2-cm-long scar (white arrow). (B) Fifteenmonth postoperative photograph. The scar (white arrow) had become lighter and no recurrence was observed on the skin.

doxycycline, steroid therapy (oral, local, or intralesional) and complete resection. Surgical treatment is usually performed for a single mass confined to the skin [2,10-13]. The patient described herein received surgical treatment, and no additional treatment was conducted because no abnormal findings were found in further tests to assess the possibility of infection and systemic invasion in consultation with the hematology-oncology department. A PET scan was performed to rule out lymphoma and systemic invasion. Since imaging showed no unusual findings, we decided to observe the patient further. The patient was followed up for 15 months without additional laboratory studies or imaging, and was monitored for complications. No specific guideline exists for the period or protocol of follow-up in such cases, and the literature also describes a variety of follow-up periods (Table 1). During follow-up, no recurrence was observed and there were no other problems that threatened the patient's survival. The reason for this patient's favorable prognosis is thought to be that the lesion was a single nodule and there was no systemic invasion. Accordingly, the authors report the diagnosis and treatment of a case of primary cutaneous CD4+ small/medium T-cell lymphoproliferative disorder, a rare disease. 
Table 1. Review of follow-up periods and protocols in published cases of primary cutaneous CD4+ small/medium T-cell lymphoproliferative disorder

\begin{tabular}{lccc}
\hline Author (year) & No. of patients & Follow-up period (mo) & Follow-up protocol \\
\hline Garcia-Herrera et al. (2008) [6] & 24 & $24^{\text {b) }}$ & \\
Grogg et al. (2008) [7] & 15 & $9^{\text {b) }}$ & \\
Beltraminelli et al. (2009) [3] & 45 & $64.3(1-357)^{\text {a) }}$ & \\
Baum et al. (2011) [10] & 10 & $24.5(5-332)^{\text {a) }}$ & \\
Alberti-Violetti et al. (2016) [8] & 61 & $36(1-191)^{\text {a) }}$ & Regular follow-up every 6 to 12 mo \\
Maurelli et al. (2017) [12] & 6 & $36(24-48)^{\text {b) }}$ & Laboratory test, lactate dehydrogenase, B2 microglobulin, imaging studies \\
& & & (ultrasonography, chest X-rays)
\end{tabular}

a)Mean (range); b)Median (range).

\section{NOTES}

\section{Conflict of interest}

No potential conflict of interest relevant to this article was reported.

\section{Ethical approval}

The study was approved for exemption by the Institutional Review Board of Konkuk University Medical Center (IRB exemption No. KUMC 2020-04-043). The requirement for informed consent was waived because of the retrospective observational character of the study.

\section{Patient consent}

The patient provided written informed consent for the publication and the use of his images.

\section{ORCID}

Jeenam Kim

Minkyoung Jeong

Dongkeun Jun

Myungchul Lee

Donghyeok Shin

Wookyoun Kim

Hyungon Choi

https://orcid.org/0000-0002-4080-6135
https://orcid.org/0000-0002-8250-0388
https://orcid.org/0000-0001-9017-9929
https://orcid.org/0000-0002-9721-0092
https://orcid.org/0000-0002-8450-4411
https://orcid.org/0000-0003-4880-5134
https://orcid.org/0000-0002-3816-1286

\section{Author contribution}

Conceptualization: JK, MJ, HC. Data curation: ML, WK, HC. Formal analysis: ML, DS. Methodology: DJ. Visualization: JK. Writing - original draft: JK, HC. Writing - review \& editing: JK, MJ, DJ, ML, DS, WK, HC. Supervision: DJ, ML, DS, HC.

\section{REFERENCES}

1. Willemze R, Cerroni L, Kempf W, Berti E, Facchetti F, Swerd- low SH, et al. The 2018 update of the WHO-EORTC classification for primary cutaneous lymphomas. Blood 2019;133:170314.

2. Swerdlow SH, Campo E, Pileri SA, Harris NL, Stein H, Siebert $\mathrm{R}$, et al. The 2016 revision of the World Health Organization classification of lymphoid neoplasms. Blood 2016;127:2375-90.

3. Beltraminelli H, Leinweber B, Kerl H, Cerroni L. Primary cutaneous CD4+ small-/medium-sized pleomorphic T-cell lymphoma: a cutaneous nodular proliferation of pleomorphic $\mathrm{T}$ lymphocytes of undetermined significance? A study of 136 cases. Am J Dermatopathol 2009;31:317-22.

4. Salah E. Primary cutaneous CD4+ small/medium pleomorphic T-cell lymphoproliferative disorder: where do we stand? A systematic review. J Dtsch Dermatol Ges 2019;17:123-36.

5. Li D, Guo B, Li D, Chang C, Lu Q. Primary cutaneous CD4+ small-to-medium-sized pleomorphic T-cell lymphoma: a rare case report of infant. J Clin Pathol 2015;68:855-8.

6. Garcia-Herrera A, Colomo L, Camos M, Carreras J, Balague O, Martinez A, et al. Primary cutaneous small/medium CD4+ Tcell lymphomas: a heterogeneous group of tumors with different clinicopathologic features and outcome. J Clin Oncol 2008; 26:3364-71.

7. Grogg KL, Jung S, Erickson LA, McClure RF, Dogan A. Primary cutaneous CD4-positive small/medium-sized pleomorphic T-cell lymphoma: a clonal T-cell lymphoproliferative disorder with indolent behavior. Mod Pathol 2008;21:708-15.

8. Alberti-Violetti S, Torres-Cabala CA, Talpur R, Corti L, Fanoni D, Venegoni L, et al. Clinicopathological and molecular study of primary cutaneous CD4+ small/medium-sized pleomorphic T-cell lymphoma. J Cutan Pathol 2016;43:1121-30.

9. Shi HZ, Zhang J, Xiong JS, Gan L, Jiang YQ, Xu XL, et al. Clinicopathological analysis of primary cutaneous CD4-positive small/medium pleomorphic T-cell lymphoproliferative disorder: a retrospective study of 22 patients. Int J Dermatol 2021; 60:497-502. 
10. Baum CL, Link BK, Neppalli VT, Swick BL, Liu V. Reappraisal of the provisional entity primary cutaneous $\mathrm{CD} 4+$ small/medium pleomorphic T-cell lymphoma: a series of 10 adult and pediatric patients and review of the literature. J Am Acad Dermatol 2011;65:739-48.

11. Toberer F, Hartschuh W, Hadaschik E. Primary cutaneous CD4+ small- to +medium-sized pleomorphic T-cell lymphoma: temporary remission by oral doxycycline. JAMA Dermatol
2013;149:956-9.

12. Maurelli M, Colato C, Gisondi P, Girolomoni G. Primary cutaneous CD4+ small/medium pleomorphic T-cell lymphoproliferative disorder: a case series. J Cutan Med Surg 2017;21:502-6.

13. Keeling BH, Gavino ACP, Admirand J, Soldano AC. Primary cutaneous CD4-positive small/medium-sized pleomorphic Tcell lymphoproliferative disorder: report of a case and review of the literature. J Cutan Pathol 2017;44:944-7. 